\title{
Carbon monoxide: from toxin to endogenous modulator of cardiovascular functions
}

R.A. Johnson ${ }^{1}$,
F. Kozma ${ }^{2}$ and
E. Colombari

\section{Correspondence}

E. Colombari

Departamento de Fisiologia

EPM, UNIFESP

Rua Botucatu, 862

04023-060 São Paulo, SP

Brasil

Fax: + 55-11-549-2127

E-mail:

colombari@ fisiocardio.epm.br

Presented at the XIII Annual Meeting of the Federação de Sociedades de Biologia Experimental, Caxambu, MG, Brasil, August 26-29, 1998.

R.A. Johnson is the recipient of a FAPESP fellowship ( $\mathrm{No}$. 97/10415-9). E. Colombari is the recipient of FAPESP and CNPq fellowships (Nos. 96/6075-5 and 520059/96-4, respectively).

Received September 17, 1998 Accepted O ctober 22, 1998

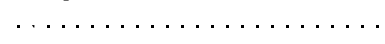

\section{Abstract}

Carbon monoxide (CO) is a pollutant commonly recognized for its toxicological attributes, including CNS and cardiovascular effects. But $\mathrm{CO}$ is also formed endogenously in mammalian tissues. Endogenously formed $\mathrm{CO}$ normally arises from heme degradation in a reaction catalyzed by heme oxygenase. While inhibitors of endogenous $\mathrm{CO}$ production can raise arterial pressure, heme loading can enhance $\mathrm{CO}$ production and lead to vasodepression. Both central and peripheral tissues possess heme oxygenases and generate $\mathrm{CO}$ from heme, but the inability of heme substrate to cross the blood brain barrier suggests the CNS heme-heme oxygenase-CO system may be independent of the periphery. In the CNS, CO apparently acts in the nucleus tractus solitarii (NTS) promoting changes in glutamatergic neurotransmission and lowering blood pressure. At the periphery, the heme-heme oxygenase- $\mathrm{CO}$ system can affect cardiovascular functions in a two-fold manner; specifically: 1) heme-derived CO generated within vascular smooth muscle (VSM) can promote vasodilation, but 2 ) its actions on the endothelium apparently can promote vasoconstriction. Thus, it seems reasonable that the CNS-, VSM- and endothelial-dependent actions of the heme-heme oxygenase-CO system may all affect cardiac output and vascular resistance, and subsequently blood pressure.

\section{Changing personalities}

Carbon monoxide is readily recognized for its toxic effects. Its colorless and odorless properties, combined with its tenacious ability to compete with oxygen for binding to hemoglobin, can lull its victims into a gradual unnoticed somnolence and vasodepression which all too often are fatal. Less appreciated is the tendency for long periods of low level carbon monoxide exposure to promote

\section{Key words}

- Carbon monoxide

- Heme

- Heme oxygenase

- Blood pressure

- Central nervous system

- Vascular tone

neural pathologies and elicit changes in cardiovascular functions $(1,2)$.

Carbon monoxide binds to hemoglobin and a minimum of $0.5-1 \%$ carboxyhemoglobin is inevitably detected in most mammalian species. While initially thought to be a manifestation of widespread environmental contamination, it was eventually realized that these persistent carboxyhemoglobin levels arise from carbon monoxide which is formed within the host. This endogenously formed 
carbon monoxide arises primarily as a product of heme metabolism (3). Until recently, endogenously formed carbon monoxide was regarded simply as a waste product, but there is now a growing awareness that even endogenously formed carbon monoxide may influence biological functions (4).

While much has been learned about the toxicological actions of carbon monoxide and about the biochemistry related to endogenous carbon monoxide formation, only recent studies have tried to functionally link these two areas. The purpose of this review is to highlight some of the important physical properties and the biochemical actions of carbon monoxide, to review some of the tools used to investigate the endogenous carbon monoxide system, and to summarize the apparent acute effects of this system as they relate to cardiovascular functions.

The toxicological aspects of carbon monoxide have been studied for decades. Diverse models of distribution kinetics, the performance of dose-time related actions, and the identification of multiple organ involvement are evidence that the actions of carbon monoxide can be quite complex (5). While the critical details of the toxicological actions of carbon monoxide are admittedly well beyond the scope of the current review, comprehensive works on the biochemical and cardiovascular effects of carbon monoxide intoxication have been published elsewhere $(1,2,5,6)$. Since the toxicology literature serves as a basis for many of the known actions of carbon monoxide, a few features culled from those studies should still be mentioned to appreciate some of the potential actions of endogenously formed carbon monoxide on biological processes.

\section{Messenger systems}

Heme proteins are often identified by their tendency to bind carbon monoxide (7). For heme proteins possessing enzymatic properties, the binding of carbon monoxide is often accompanied by changes in their metabolic activities (7-10). Accordingly, any or all heme proteins would seem particularly well suited to be potentially involved in the biological actions of carbon monoxide.

Soluble guanylate cyclase is a commonly considered candidate $(4,8)$. Carbon monoxide activates purified soluble guanylate cyclase to increase cGMP formation approximately two-fold, which is relatively modest when compared to the 100-fold greater increases that may be obtained by activation with nitric oxide (8). However, such differences in efficacy do not preclude the possibility that functional effects may still arise from the increases in cGMP production which can be attained from carbon monoxide-induced activation of soluble guanylate cyclase.

Carbon monoxide was shown to dilate vascular smooth muscle preparations in a manner which was prevented by methylene blue, an inhibitor of soluble guanylate cyclase (11). Consequently, it was suggested that carbon monoxide-induced vasodilation of vascular smooth muscle was soluble guanylate cyclase-dependent. But recent studies employing newly developed inhibitors of soluble guanylate cyclase have shown that carbon monoxide-induced dilation may proceed even when soluble guanylate cyclase activity has been blocked $(12,13)$. These findings suggest that carbon monoxide, at least under some conditions, may promote relaxation of vascular smooth muscle by processes which are soluble guanylate cyclase-independent.

The cytochrome P-450 family of enzymes (cytP450), which collectively display a spectral shift in response to carbon monoxide binding, are another class of enzymes proposed as potential mediators of carbon monoxide-induced effects (7). However, it has been suggested that the $\mathrm{CO} / \mathrm{O}_{2}$ ratio required to produce significant binding to most types of cytP450 enzymes would also be likely to overwhelm the $\mathrm{O}_{2}$-carrying capacity of cir- 
culating hemoglobin to a fatal extent $(2,5)$.

In contrast to other P450 enzymes, the activities of some mitochondrial cytochrome oxidases may be affected by levels of carbon monoxide which fall short of producing fatal hypoxia (5). Also, the binding of carbon monoxide to these cytochrome oxidases is apparently further enhanced during increased metabolic demand and in environments of reduced oxygen tensions. As these cytochromes of the mitochondrial membrane are involved in electron transport and in the generation of ATP, disruption of the associated electron shuttling may in turn lead to multiple functional effects by interfering with kinase- and other ATP-dependent processes.

Prostaglandin G/H synthase (14), catalases (15), and potassium channels (16) are also amongst the suggested potential targets for the actions of carbon monoxide. In addition, cyclooxygenase- 2 has recently been recognized to be heme-bearing (17), and thus may also participate in the biological actions of carbon monoxide. The relative contributions of each of these systems to the biological effects of carbon monoxide, if any, have yet to be fully understood.

\section{Endogenous sources of carbon monoxide and their regulation}

Endogenously formed carbon monoxide arises primarily from the degradation of monomeric free heme and is generated in a reaction catalyzed by the heme oxygenases. During this reaction heme is degraded to release equimolar quantities of iron, biliverdin and carbon monoxide (3). The iron is primarily recycled into the formation of new heme while the biliverdin is rapidly converted to bilirubin by an abundance of biliverdin reductases (18). The carbon monoxide product eventually binds to circulating hemoglobin and is then transported as carboxyhemoglobin until it is excreted through ventilation. Heme oxygenase activity in some tissue homogenates is sufficient to generate
2 nmol of carbon monoxide/mg protein per hour (19). Given that $1 \%$ carboxyhemoglobin levels in the blood are quite common, this constitutes a minimum load of approximately $100 \mu \mathrm{mol} / \mathrm{l}$ carbon monoxide trapped within the vascular space.

Normally, endogenously formed carbon monoxide arises almost exclusively from the metabolism of heme by heme oxygenase. At least three isoforms of heme oxygenase have been identified $(3,20)$ which originate from multiple mRNAs $(21,22)$. Some heavy metals, metalloporphyrins, as well as heme, have been shown to chronically induce heme oxygenase-1 while having relatively little effect on the expression of heme oxygenase-2 (21). Very little is known about the recently discovered heme oxygenase- 3 isoform (20). The regulation of heme oxygenase expression is quite complex (21), but it is clear that the induction of heme oxygenase in some tissues may allow them to participate in the local generation of carbon monoxide where they otherwise might be limited by a deficiency of the mediating enzyme.

Increased expression of heme oxygenase protein is not the only means for stimulating the generation of carbon monoxide and other heme oxygenase products. Alternatively, heme loading can rapidly increase plasma bilirubin (23) in a manner which precedes discernable induction of the enzyme. Since the provision of the substrate rapidly increases the generation of heme oxygenase products, it is clear that heme availability can be a rate-limiting step for the endogenous production of carbon monoxide.

Biochemical evidence derived from studies of delta aminolevulinic acid (dALA) synthase and its feedback inhibition by heme (24) imply that normal resting levels of free heme may be in the vicinity of $0.5-1.0 \mu \mathrm{mol} /$ 1. Oddly, heme loading in 50-fold excess of these levels typically increases plasma bilirubin levels and $\mathrm{CO}$ production by only $2-$ 3 -fold (23). The reason for these divergent trends may be related to the physical proper- 
ties of heme. In aqueous solutions at physiological $\mathrm{pH}$, monomeric free heme has been characterized as reaching saturation at around $2 \mu \mathrm{mol} / \mathrm{l}(25)$. Heme which is loaded beyond this limit progressively forms dimeric structures and polymeric chains (25). While these dimeric/polymeric forms may potentially constitute "heme stores", they apparently do not function as substrates for heme oxygenase $(3,26)$. Consequently, it can make sense that heme loading might only double or triple the concentration of monomeric free heme in tissues. In turn, this increase in heme oxygenase substrate availability should be paralleled by increases in carbon monoxide/ bilirubin formation (23).

Endogenously formed carbon monoxide does not arise solely from the degradation of heme. In fact, inhibition of heme oxygenase activity reduces carbon monoxide production by only $30-50 \%$ at best (27). While heme degradation is usually the primary source of endogenously formed carbon monoxide, the blockade of heme oxygenase activity initiates the generation of carbon monoxide from alternative sources including a process involved in fatty acid metabolism $(27,28)$. The mechanisms underlying these shifts in carbon monoxide sources are poorly understood.

The suggestion that endogenously formed carbon monoxide might participate in biological functions was highlighted in a seminal work by Gerald Marks (4). Noting that carbon monoxide is an activator of soluble guanylate cyclase, and that carbon monoxide is endogenously formed as part of heme metabolism, Marks proposed that heme-derived carbon monoxide might function in a manner synonymous to that of nitric oxide. Indeed both systems produce low molecular weight gasses which are activators of soluble guanylate cyclase, and the catalytic formation of both gases are NADPH-dependent $(3,29)$.

But the nitric oxide- and carbon monoxide-generating systems also exhibit some very important differences. Nitric oxide is highly labile, exhibiting a biological half-life of seconds (29), whereas carbon monoxide is unusually stable and highly resistant to transformation $(1,2)$. As mentioned earlier, nitric oxide is a powerful activator of soluble guanylate cyclase, whereas carbon monoxide is a weak activator of this cGMP-generating enzyme (8). Under most conditions the constitutive forms of nitric oxide synthase are primarily found in neurons and endothelium (29) but are relatively absent from vascular smooth muscle. While the constitutive isoform of heme oxygenase is similarly prevalent in neuronal and endothelial tissue domains (30), it differs from its nitric oxidegenerating counterpart by being abundant within the vascular smooth muscle. Since heme oxygenase is abundant in the smooth muscle domain of most blood vessels, conditions which promote endothelial damage or dysfunction may potentially disrupt some aspects of the nitric oxide-generating system while leaving the heme-derived carbon monoxide-generating system intact.

\section{Studying the heme-heme oxygenase- carbon monoxide system: tools and approaches}

\section{Chronic heme oxygenase induction}

Induction of heme oxygenase- 1 protein is often accompanied by an array of "heat shock" stress-related proteins and functional derangements $(21,30)$. Heavy metal salts and other stress-related events are often used to induce expression of heme oxygenase-1 protein and promote heme oxygenase activity $(30,31)$. While such chronic inductions may reflect potential pathological states which could lead to persistent elevations in endogenous carbon monoxide production, this technique may be less than optimal to distinguish the actions of heme oxygenase-derived carbon monoxide from those of the other simultaneously expressed heat-shock proteins. 


\section{Acute manipulations of the heme-heme oxygenase-carbon monoxide system}

Studies which focus on the functional effects of endogenously formed carbon monoxide may alternatively be approached by increasing and decreasing tissue levels of acutely endogenously formed carbon monoxide. Unfortunately, no selective chelators or metabolizing agents are currently available to inactivate carbon monoxide once it has been formed. But the formation of hemederived carbon monoxide can be increased by providing heme oxygenase substrate $(3,23,26)$ or conversely decreased by using metalloporphyrins that compete with heme for binding to the heme oxygenase enzyme $(13,19,21,23,24,27)$. Such a strategy has been used to identify heme-induced effects which arise from formation of a heme oxygenase product $(19,23)$. Specifically, heme-induced effects which can be prevented and/or reversed by an inhibitor of heme oxygenase are likely to be consequent to the formation of a heme oxygenase product. As iron, biliverdin and carbon monoxide are the metabolic products of heme oxygenase-mediated metabolism of heme $(3,21)$, the actions of these three products may in some cases be compared to identify the specific product which is most likely to account for the heme oxygenase-induced response.

The use of heme to drive heme oxygenase activity in experimental settings is complicated by its tendency to aggregate in aqueous solutions at physiological $\mathrm{pH}(25,32)$ and to degrade during storage (32). An acetic environment may in some cases be used to increase porphyrin solubility, but such conditions may also damage porphyrin integrity (25). Alternatively, bases such as Tris and sodium hydroxide have been used to increase the solubility of porphyrins, but these caustic solvents may also promote substantial tissue damage. Some porphyrins are soluble in ethanol, but ethanol can alter porphyrin metabolism by driving the synthesis of dALA (24), the rate-limiting step in the formation of new heme. While disodium carbonate has been used to solubilize hematin for clinical applications (24), the resulting preparation is still susceptible to degradation during storage (32).

The solubility limitations associated with heme can easily be overcome by dissolving it in an amino acid solution. Heme-L-arginate is one such commercially available preparation $(32,33)$ which is used in the treatment of porphyrin metabolic disorders (24). This preparation boosts the solubility of heme to $38 \mathrm{mmol} / \mathrm{l}$, maximizes monomeric heme availability, and yields a preparation which can be stored for months without discernible degradation (32). For some experimental protocols, heme-L-arginate may create methodological problems, since L-arginine is a substrate for nitric oxide synthase-mediated formation of nitric oxide (29). Alternatively, an arginine-free preparation of heme-Llysinate can be made by replacing L-arginine with L-lysine (32). Since heme-L-arginate and heme-L-lysinate each demonstrate heme oxygenase substrate activities identical to that of hematin (32), these preparations seem particularly well suited as sources of heme for experimental purposes.

Metalloporphyrins may be used to inhibit endogenous formation of carbon monoxide. These pharmacological tools resemble heme in their porphyrin structure, but the iron core is substituted by a heavy metal such as $\mathrm{Zn}$, $\mathrm{Sn}, \mathrm{Cr}, \mathrm{Co}$ or $\mathrm{Cu}(19,21,27,30,34)$. The tendency for some of these substituted porphyrins to compete with heme for binding to the heme oxygenase enzyme has led to their use as heme oxygenase inhibitors. Accordingly, such inhibitors can also be used to inhibit the formation of heme-derived carbon monoxide (27).

Photolability is a serious problem with some metalloporphyrins. Some metalloporphyrins, most notably those which are tinbearing, can undergo non-enzymatic degradation to release carbon monoxide when 
they are exposed to light (35). Concerns have also been raised over the potential interactions of metalloporphyrins with soluble guanylate cyclase $(36,37)$. It is abundantly clear that the substituted metalloporphyrins do not participate in the activation of soluble guanylate cyclase in a manner similar to heme (36), but demonstrations that these substituted metalloporphyrins produce functional effects which arise from their direct action on soluble guanylate cyclase have not been forthcoming.

Carbon monoxide is an obvious tool used to confirm that a heme-induced effect may be attributed to the endogenously formed carbon monoxide product. An array of techniques for preparing and administering carbon monoxide have been explored (1). For in vitro studies, carbon monoxide is water soluble and thus can be easily prepared in physiological buffer solutions. In contrast, administration of carbon monoxide for in vivo studies can be substantially complicated by its distribution kinetics which are markedly influenced by the affinity of this gas for heme proteins $(1,2)$. Carbon monoxide has been administered through inhalation (1), but the delivery of carbon monoxide to the tissues by this method is reliant on its delivery as carboxyhemoglobin. This means that carbon monoxide must bind to hemoglobin before it can be delivered to the tissues. In turn, this event produces hypoxia that must precede appreciable accumulations of carbon monoxide in the tissues. Intravenous administration of carbon monoxide solutions is equally disadvantageous because of this complication.

The intraperitoneal administration of carbon monoxide may offer some advantages. Using this simple and efficient technique, marked changes in cardiovascular function can precede dramatic changes in carboxyhemoglobin levels (38). When carbon monoxide gas is injected into the peritoneum, its appearance in the vasculature as carboxyhemoglobin is not immediate and in some spe- cies may not plateau for hours (2). The delayed transit of this water soluble and low molecular weight gas from the peritoneum to the vascular space strongly suggests that the extravascular tissues also possess a marked affinity for carbon monoxide. Furthermore, it supports what toxicologists have emphasized for years, that carboxyhemoglobin levels are of limited value when judging the carbon monoxide content of the extravascular space $(1,2)$.

\section{The heme-heme oxygenase-carbon monoxide system and cardiovascular functions}

Since blood pressure is calculated as the product of cardiac output and total peripheral resistance, cardiovascular functional studies accordingly focus on these determinants. Based upon the toxicological literature and the logical rationale proposed by Gerald Marks $(2,4,8)$, it appears that heme-derived carbon monoxide might serve as a vasodilator and accordingly tend to be vasodepressive.

\section{Chronic blood pressure effects of the heme- heme oxygenase-carbon monoxide system}

Volumes have been published on various aspects of heme oxygenase induction $(21,30)$. Studies preceding the overt suggestion that endogenous carbon monoxide may participate in biological functions showed that inducers of heme oxygenase such as $\mathrm{SnCl}_{2}$ could chronically slow the development of hypertension in spontaneously hypertensive rats (SHR) (31). Since the vasodepressive effects were accompanied by decreases in microsomal cytochrome content, those early studies attributed the blood pressure lowering effects of heme oxygenase induction to a state of heme depletion and a consequent reduction of the formation of cytP450-derived vasoconstrictive eicosanoids (31). While this explanation was consistent with the findings, those corollary studies did not 
directly verify a state of free heme depletion. It was then also shown that chronic heme loading could induce heme oxygenase and similarly slow down the development of hypertension in SHRs (39). Since monomeric free heme is the substrate for heme oxygenase, and since heme loading is known to increase bilirubin production, this suggests that heme substrate availability is not impaired by these treatments. Rather, it supports the idea that a heme oxygenase product such as carbon monoxide may be involved in the vasodepressive effect.

Certainly such evidence does not preclude a role for cytP450-derived eicosanoids in heme oxygenase-induced vasodepression. Indeed, changes in cytP450 and heme oxygenase content accompany a variety of chronic disease states $(21,30,31)$. Nonetheless, studies which rely solely on chronic corollaries between induction and functions are not able to distinguish the effects of heme oxygenase activity from those of the other simultaneously expressed heat-shock proteins. Interpretations of studies using chronic treatment of heme oxygenase inhibitors are similarly complicated as such inhibitors tend to induce heme oxygenase along with other heat-shock proteins $(21,31)$, while at the same time directly inhibiting heme oxygenase. In addition, these chronic treatments may potentially produce effects by altering other less investigated functional aspects of porphyrin metabolism-dependent processes such as dALA synthase activity or glutathione metabolism(24). Furthermore, chronic inhibition of heme oxygenase activity through the use of metalloporphyrins or even highly specific antibodies may have limited influence on total carbon monoxide production as sources of nonheme-derived carbon monoxide may be greatly amplified during such conditions (27).

\section{Acute blood pressure effects of the heme- heme oxygenase-carbon monoxide system}

The use of heme oxygenase substrates and inhibitors to acutely manipulate heme oxygenase activity constitutes a major development for studying the biological actions of the heme-heme oxygenase-carbon monoxide system. This strategy optimizes the ability to examine heme oxygenase-related effects in a setting which maximally precedes potential enzyme induction and shifting in carbon monoxide sources. By so doing, these inhibitors of heme oxygenase may also be used to evaluate the contributions of the heme-heme oxygenase-carbon monoxide system to basal functions.

Systemic administration of metalloporphyrins which inhibit heme oxygenase can rapidly produce vasoconstriction and raise blood pressure in rats (40). This suggests that a heme oxygenase product serves to reduce vascular tone. In contrast, heme preparations that drive heme oxygenase-mediated formation of carbon monoxide, such as hemeL-lysinate, lower blood pressure in chronically hypertensive SHR and DOCA-salt rat models and in rats made acutely hypertensive by phenylephrine infusion (23). These heme-induced decreases in blood pressure can be prevented by pretreatment with inhibitors of heme oxygenase. This suggests that the vasodepressive actions of heme are consequent to the formation of a heme oxygenase product.

It is well established that the heme oxygenase-mediated degradation of heme forms three products: iron, biliverdin and carbon monoxide (3). Iron chelation does not prevent heme-induced vasodepression in SHRs (23), and biliverdin does not acutely promote vasodepression in either normotensive or hypertensive models $(23,40)$. However, intraperitoneal injection of carbon monoxide does preferentially lower blood pressure in hypertensive models (23). Thus, it appears that the heme-induced vasodepression is more likely to arise from the formation of the carbon monoxide product.

Hypertensive models do seem to display an enhanced sensitivity for heme-induced vasodepression (23). While this might sug- 
gest that the hypertensive models such as the SHRs are heme-, heme oxygenase-, or carbon monoxide-deficient compared to normotensive Sprague-Dawleys, these two strains do not differ in plasma bilirubin content (23). Furthermore, matched loadings with heme preferentially lower blood pressure in the hypertensive models despite similar 2-3-fold elevations in the plasma bilirubin content in both normotensive and hypertensive strains. A variety of hypertensive models have been found to be both acutely (23) and chronically (1) more sensitive to the vasodepressive actions of exogenously applied carbon monoxide than their normotensive counterparts. These tendencies indicate that these normotensive and hypertensive models do not appear to differ in their abilities to generate heme oxygenase products, including carbon monoxide. Rather they suggest that SHRs, and perhaps other hypertensive models as well, may be more sensitive to the vasodepressant actions of the carbon monoxide product.

\section{CNS involvement in heme oxygenase-related cardiovascular effects}

Heme oxygenase and its activity have been identified in the brain $(21,40-42)$, but its distribution is such that it forms distinct tracts (41). While some metalloporphyrins like ZnDPBG have been designed to pass the blood brain barrier to inhibit heme oxygenase activity in the brain $(27,40)$, studies using radiolabeled tags show that heme does not pass the blood brain barrier (33). In addition, dALA synthase, the rate-limiting enzyme in the formation of new heme, and the other enzymes involved in heme synthesis have been identified in brain tissues (24). The finding that the brain is insulated from infiltration by circulating heme but locally possesses the metabolic processes for heme synthesis suggests that the CNS may constitute a heme oxygenase functional unit which is distinct from that of peripheral tissues.
Intraperitoneal administration of ZnDPBG can inhibit heme oxygenase activity both at the periphery and in the brain, decrease the systemic production of carbon monoxide (27), and increase the blood pressure of the awake rat in a manner which is paralleled by an increase in total peripheral resistance (40). This pressor response can be prevented by alpha-1-adrenergic receptor antagonists or pharmacological blockade of ganglionic function, but not by bilateral sino-aortic deafferentation. These findings suggest that ZnDPBG-induced elevations in blood pressure may involve a heme oxygenase-related action which is central $(40,43)$.

The nucleus tractus solitarius (NTS) is the site of termination of cardiovascular and respiratory afferent fibers in the medulla oblongata (44) and is a key structure in glutamatergic-mediated cardiovascular afferents and an integrative region of baroreceptor and chemoreceptor responses (45). Systemic inhibition of heme oxygenase has been shown to attenuate the pressure-bradycardia relationship, which is modulated via the NTS (43). Inhibitors of heme oxygenase can affect glutamatergic-mediated depolarization in isolated NTS preparations (46). Since isolated NTS tissues respond to manipulations of the heme-heme oxygenase-carbon monoxide system, and since the NTS is involved both in the modulation of baroreceptor reflex activity and in the establishment of blood pressure, it seems that the NTS might be involved in the ZnDPBG-induced pressor response.

This notion is supported by the observation that microinjections of ZnDPBG into the NTS are able to increase blood pressure in a manner that can be reversed by microinjections of carbon monoxide (43). Likewise, microinjections of carbon monoxide into the NTS can reverse the elevation in blood pressure produced by systemic administration of ZnDPBG (43). Thus it appears that the heme oxygenase product formed in the NTS exerts a vasodepressive influence; it also appears 
that the heme oxygenase product is likely to be carbon monoxide.

L-Glutamate is a neurotransmitter involved in NTS-mediated afferent baroreceptor activity. NTS microinjections of heme oxygenase inhibitors do attenuate the pressor responses to microinjections of L-glutamate (47) and interfere with baroreceptor activity. Such findings suggest that heme metabolism may locally interact with glutamatergic transmission in the NTS. Even so, it remains unclear if these glutamatergiclinked effects arise solely from its influences on carbon monoxide product formation.

While evidence is mounting to suggest neuromodulatory roles for heme oxygenasederived carbon monoxide, special caution should be observed when designing and interpreting CNS studies using metalloporphyrins. The potential of metalloporphyrin and heme to affect the CNS independently of carbon monoxide generation may represent a highly complicated topic. In the porphyrin metabolic pathway, the formation of dALA is enzymatically mediated and constitutes the rate-limiting step in the formation of new heme (24). In turn, the concentration of free heme serves as a feedback inhibitor of dALA synthase activity. This is the mechanism by which tissue levels of heme are effectively maintained. This heme precursor, dALA, is structurally related to gamma aminobutyric acid (GABA). In addition, heme itself demonstrates affinity for benzodiazepine receptors. It is accepted that GABA and benzodiazepine receptor functions are cooperative and may play important roles in central regulation of blood pressure. While the effects of dALA on GABA receptors and the effects of heme on benzodiazepine receptor functions have not been fully characterized, it should be mentioned that any maneuver which influences heme availability may also potentially affect benzodiazepine- and GABArelated functions in the CNS.

Acute intact animal studies which use systemic administration of heme are likely to be less complicated by CNS-related effects since heme does not cross the blood brain barrier (33). However, inhibitors of heme oxygenase which cross the blood brain barrier (40), and carbon monoxide as well $(1,2)$, can potentially affect the blood pressure by acting directly on the NTS or other central structures. Since the CNS exerts a powerful influence on cardiovascular functions, the actions of the heme-heme oxygenase-carbon monoxide system directly on vascular activity may be best addressed by using isolated vessel preparations which are devoid of central and circulating influences.

\section{Endogenous carbon monoxide and vascular tone: dual actions}

\section{Carbon monoxide as a vasoconstrictor}

Heme oxygenase has been identified both in arteriolar and venular smooth muscle, and has also been located in arteriolar endothelium (48). The findings that carbon monoxide can stimulate soluble guanylate cyclase (8) and relax preparations of vascular smooth muscle (11) have prompted suggestions that carbon monoxide may lower blood pressure by promoting vasodilation (4). In contrast, earlier studies $(7,49-52)$ have provided ample evidence that carbon monoxide can bind to nitric oxide synthase and inhibit the production of nitric oxide, a vasodilator. If so, it might be expected that carbon monoxide could block basal nitric oxide production and cause vasoconstriction with a consequent increase in blood pressure. While these two functionally opposing roles of endogenously formed carbon monoxide in vascular tone are equally plausible, several studies using isolated vessels and perfused preparations $(7,53-56)$ have suggested that hemederived carbon monoxide may be vasodilatory. Such reports have understandably typified carbon monoxide as a vasodilator.

What was lacking was a strategic attempt to elucidate the endothelial-dependent roles 
of endogenously formed carbon monoxide from those which are endothelium-independent. In an attempt to clarify this issue, we recently found that heme can elicit vasodilation of isolated superfused rat gracilis firstorder arterioles denuded of endothelium, in a manner which is readily reversed by an inhibitor of heme oxygenase (57). In that study, carbon monoxide similarly elicited vasodilation in the preparations denuded of endothelium. These observations suggest that a heme oxygenase product, apparently carbon monoxide, can produce vasodilation of these resistance vessels in a manner which is endothelium-independent. It also suggests that the vasodilatory actions of heme can be independent of the generation of endothelium-derived factors.

In sharp contrast, we found that heme elicited a vasoconstriction in similarly prepared arterioles when the endothelium was left intact (57). In addition, we found that the heme-induced vasoconstriction was readily reversed by the further inclusion of an inhibitor of heme oxygenase. Furthermore, in similarly prepared vessels in which the endothelium was left intact, we found that exogenously applied carbon monoxide could also elicit a vasoconstrictive response. These frankly unexpected findings suggested that a heme oxygenase product, which was mimicked by carbon monoxide, could also elicit vasoconstriction by 1) facilitating the release of a vasoconstrictive agent from the endothelium or by 2) interfering with the release of an endothelium-derived relaxing factor.

Nitric oxide is an endothelium-derived relaxing factor (29). In addition, nitric oxide synthase-mediated formation of nitric oxide has been repeatedly shown to be inhibited by carbon monoxide $(7,49-52)$. If the observed endothelium-dependent HLL-induced vasoconstriction of the gracilis arteriole preparations were a consequence of inhibition of nitric oxide synthase activity by endogenously formed carbon monoxide, it would seem reasonable that the blockade of nitric oxide formation should allow the vasodilatory influences of heme-derived carbon monoxide to predominate.

In vessels with intact endothelium and in which L-NAME is present to maximally inhibit nitric oxide synthesis, we find that heme does elicit vasodilation in a manner which is reversed by inhibitors of heme oxygenase $(12,53,57)$. Blockade of nitric oxide synthases similarly allows the vasodilatory effects of carbon monoxide to be displayed even when the endothelium is present $(12,53,57)$. Accordingly, the simplest explanation is that heme-derived carbon monoxide, specifically that formed in smooth muscle, may serve to promote vasodilation by a local action. Simultaneously these vasodilatory actions may be buffered by the consequent withdrawal of the vasodilatory influences of endothelium-derived nitric oxide.

While such observations are consistent with the well-documented biochemical actions of carbon monoxide to block nitric oxide synthase, they are admittedly in sharp contrast to the recent dogma which highlights the vasodilatory actions of carbon monoxide. Coceani et al. (54) have reported that vasodilation can be elicited by heme substrate in intact lamb ductus arteriosus vessels. Such findings may seem in contrast to our recent findings, but they have carefully documented that heme oxygenase is localized in the smooth muscle domain of their ductus vessels, but not present in the endothelium.

Given the widely accepted importance of the nitric oxide system for multiple biological processes (29), the possibility of a carbon monoxide/nitric oxide interaction should be explored to determine its hemodynamic functional significance. Consideration of this potential interaction is crucial, since ignorance of this feature might seriously confound the interpretations of key experiments in this newly emerging area of endogenous carbon monoxide research. 


\section{Vasodilatory influences of endogenous carbon monoxide}

While we recently suggested that carbon monoxide may promote vasoconstriction by interfering with the vasodilatory influences of the nitric oxide system, that suggestion was certainly not intended to overlook the vasodilatory influences of endogenously formed carbon monoxide. Given the apparent effects of carbon monoxide attenuating the vasodilatory influences of the nitric oxide vasodilatory system, the vasodilatory effects of heme can be optimized by using preparations in which the nitric oxide system is inhibited.

In isolated superfused rat gracilis firstorder arterioles with intact endothelium, in an environment containing L-NAME to maximally inhibit nitric oxide synthase, heme does promote vasodilation in a manner which is readily reversed by inhibitors of heme oxygenase (53). This suggests the hemeinduced vasodilatory response arises from the generation of a heme oxygenase product. These heme-induced vasodilatory effects are not prevented by an iron chelator or mimicked by biliverdin. However, carbon monoxide does mimic this heme-induced dilation of similar preparations. This is the evidence that the vasodilatory heme oxygenase product is most likely to be carbon monoxide.

In similarly prepared resistance vessels, in which the nitric oxide system has been maximally inhibited, inhibitors of heme oxygenase alone produce a striking vasoconstriction (12). This vasoconstrictive effect can be both prevented and reversed by carbon monoxide, but cannot be prevented by a nitric oxide donor. These findings suggest that the basal production of carbon monoxide may exert a substantial vasodilatory influence on these resistance vessels.

Concerns have recently surfaced over potential interactions between metalloporphyrins and soluble guanylate cyclase (36,
37). However, the arteriolar constriction produced by chromium mesoporphyrin is unaffected by an inhibitor of soluble guanylate cyclase (12). Furthermore, vasodilatory actions of exogenously applied carbon monoxide or heme also appear to be unaffected by an inhibitor of soluble guanylate cyclase in isolated arteriolar preparations (12). Accordingly, it appears that heme, at least in some conditions, carbon monoxide and metalloporphyrins can produce effects which are unrelated to soluble guanylate cyclase activity. While the mechanisms underlying acute carbon monoxide-induced relaxation of vascular smooth muscle have yet to be clarified, they apparently arise independently from P450 -, endothelin- or soluble guanylate cyclase-related activities (12).

\section{Proposed organization for endogenously derived carbon monoxide effects on cardiovascular functions}

An abundance of literature has accumulated describing intricate details of mechanisms which contribute to heme oxygenase expression. There has also been a virtually endless trail of proposed biochemical interactions of carbon monoxide and heme metabolites with almost every imaginable messenger system. While such information is certainly of tremendous importance, there may have been a tendency for the biochemistry to overwhelm the functional effects of this newly explored system. The identification of the primary functions of endogenously formed carbon monoxide may serve as a point of orientation in the search to identify the underlying primary biochemical and molecular biological mechanisms. By such an approach, the physiological contributions of the heme-heme oxygenase-carbon monoxide system may best be appreciated.

The current review has preferentially emphasized the apparent functional consequences of the heme-heme oxygenase-car- 
bon monoxide system in an attempt to organize a framework to identify the primary influences of endogenously formed carbon monoxide on cardiovascular functions. What seems to be emerging are multiple mechanisms by which endogenously formed carbon monoxide exerts effects on cardiovascular control.

Specifically, heme-derived carbon monoxide generated within vascular smooth muscle appears to be vasodilatory. This vasodilatory influence may be countered by carbon monoxide-induced withdrawal of the endothelium-dependent vasodilatory influence of the nitric oxide system. This suggests that under some conditions carbon monoxide may have little direct effect on basal vascular tone. Even so, such conditions could still interfere with the dynamic influences related to shear force-mediated changes of the nitric oxide system and consequently amplify the vasoactive actions of other agents. Such an arrangement means that carbon monoxide, even the endogenously formed one, may promote a propensity for vasospasms. Since carbon monoxide has been shown to compete with L-arginine for binding to nitric oxide synthase, then L-arginine may potentially protect nitric oxide synthase from the inhibitory actions of carbon monoxide. It should be emphasized that it is not clear to what extent the nitric oxide and carbon monoxide systems interact in vivo. Nonetheless, potential interactions between the carbon monoxide- and nitric oxide-generating systems should be seriously considered, especially when isolated preparations are involved.

In addition to the proposed endotheliumdependent and -independent actions of carbon monoxide on vascular tone, it appears that carbon monoxide can affect blood pressure through CNS-mediated influences on vascular tone and baroreceptor reflex functions. One region in the brain that has been strongly implicated as participating in these functions is the NTS. In some cases, the actions of the CNS may exert a third influence which "tips the balance" of the directly offsetting endothelium-dependent and -independent actions of carbon monoxide on the vasculature.

While the search for functional roles for the heme-heme oxygenase-carbon monoxide system remains in its infancy, the accrued evidence strongly suggests that the heme metabolic system is doing more than simply generating carbon monoxide as a waste product. More specifically, the evidence suggests that heme-derived carbon monoxide may exert effects on blood pressure by influencing both endothelium-dependent and -independent functions in the vasculature, and these periferal effects may be further adjusted by carbon monoxideinduced influences arising through the central nervous system.

\section{References}

1. Penney DG (1988). A review: Hemodynamic response to carbon monoxide. Environmental Health Perspectives, 77: 121130.

2. Penny DG (1996). Carbon Monoxide. 1st edn. CRC Press, Inc., New York.

3. Tenhunen R, Marver HS \& Schmid R (1969). Microsomal heme oxygenase. J ournal of Biological Chemistry, 244: 6388-6394.

4. Marks GS, Brien JF, Nakatsu K \& McLaughlin BE (1991). Does carbon mon- oxide have a physiological function? Trends in Pharmacological Sciences, 12: 185-188.

5. Raub J A \& McMullen TB (1991). Air Quality Criteria for Carbon Monoxide. 1st edn. United States Environmental Protection Agency, Washington, DC.

6. Penney DG (1990). Acute carbon monoxide poisoning: animal models: a review. Toxicology, 62: 123-160.

7. White KA \& Marletta MA (1992). Nitric oxide synthase is a cytochrome P-450 type hemoprotein. Biochemistry, 31 : 6627-6631.

8. Schmidt HHHW (1992). NO, CO and HO endogenous soluble guanylyl cyclase-activating factors. FEBS Letters, 307: 102107.

9. Raff H \& J ankowski B (1994). Inhibition of aldosterone release by hypoxia in vitro: interaction with carbon monoxide. J ournal of Applied Physiology, 76: 689-693.

10. Wada A, Ohnishi T, Nonaka Y, Okamoto M \& Yamano T (1985). Synthesis of aldos- 
terone by a reconstituted system of cytochrome P-45011 beta from bovine adrenocortical mitochondria. J ournal of Biochemistry, 98: 245-256.

11. Graser T, Vedernikov YP \& Li DS (1990). Study on the mechanism of carbon monoxide induced endothelium-independent relaxation in porcine coronary artery and vein. Biochimica et Biophysica Acta, 49: 293-296.

12. Kozma F, J ohnson RA, Tong $X \&$ Nasjletti A (1998). Role of endogenous carbon monoxide in the regulation of basal tone in resistance vessels. Hypertension, 32: 60 (Abstract).

13. Coceani F, Kelsey L \& Seidlitz E (1996). Carbon monoxide-induced relaxation of the ductus arteriosus in the lamb: evidence against the prime role of guanylyl cyclase. British J ournal of Pharmacology, 118: 1689-1696.

14. Gaspard S, Chottard G, Mahy J P \& Mansuy D (1996). Study of the coordination chemistry of prostaglandin $\mathrm{G} / \mathrm{H}$ synthase by resonance Raman spectroscopy. European J ournal of Biochemistry, 238: 529-537.

15. Hu S \& Kincaid J R (1992). Resonance Raman studies of the carbonmonoxy form of catalase. Evidence for and effects of phenolate ligation. FEBS Letters, 314: 293-296.

16. Wang $R$, Wu $L \&$ Wang $Z$ (1997). The direct effect of carbon monoxide on $\mathrm{KCa}$ channels in vascular smooth muscle cells. Pfluegers Archives, 434: 285-291.

17. Percival MD, Ouellet $M$, Vincent $C$ J , Yergey J A, Kennedy BP \& O'Neill GP (1994). Purification and characterization of recombinant human cyclooxygenase- 2 . Archives of Biochemistry and Biophysics, 315: 111-118.

18. Kutty RK \& Maines MD (1984). Hepatic heme metabolism: Possible role of biliverdin in the regulation of heme oxygenase activity. Biochemical and Biophysical Research Communications, 122: 40-46.

19. Cook MN, Nakatsu K, Marks GS, McLaughlin BE, Vreman $H J$, Stevenson DK \& Brien J F (1995). Heme oxygenase activity in adult rat aorta and liver as measured by carbon monoxide formation. Canadian J ournal of Physiology and Pharmacology, 73: 515-518.

20. McCoubrey J r WK, Huang TJ \& Maines MD (1997). Isolation and characterization of a cDNA from the rat brain that encodes hemeprotein heme oxygenase-3. European J ournal of Biochemistry, 247: 725732.

21. Maines MD (1997). The heme oxygenase system: A regulator of second messenger classes. Annual Reviews in Pharmacology and Toxicology, 37: 517-554.

22. McCoubrey J r WK, Eke B \& Maines MD (1995). Multiple transcripts encoding heme oxygenase- 2 in rat testis: developmental and cell-specific regulation of transcripts and protein. Biology of Reproduction, 53: 1330-1338.

23. J ohnson RA, Lavesa M, DeSeyn $K$, Scholer MJ \& Nasjletti A (1996). Heme oxygenase substrates acutely lower blood pressure in hypertensive rats. American J ournal of Physiology, 271: H1132-H1138.

24. Moore MR, McColl KEL, Rimington $C \&$ Goldberg A (1987). Disorders of Porphyrin Metabolism. Plenum Press, New York.

25. FalkJ E (1964). Porphyrins and M etalloporphyrins. Elsevier Publishing Company, Amsterdam, Holland.

26. Tenhunen R (1972). Method for microassay of microsomal heme oxygenase activity. Analytical Biochemistry, 45: 600-607.

27. Vreman $\mathrm{HJ}$, Lee $\mathrm{OK} \&$ Stevenson DK (1991). In vitro and in vivo characteristics of a heme oxygenase inhibitor: ZnBG. American J ournal of the Medical Sciences, 302: 335-341.

28. Wolff DG \& Bidlack WR (1976). The formation of carbon monoxide during peroxidation of microsomal lipids. Biochemical and Biophysical Research Communications, 73: 850-857.

29. Moncada S \& Higgs EA (1995). Molecular mechanisms and therapeutic strategies related to nitric oxide. FASEB J oumal, 9: 1319-1330.

30. Maines MD (1988). Heme oxygenase: function, multiplicity, regulatory mechanisms, and clinical applications. FASEB J ournal, 2: 2557-2568.

31. Sacerdoti D, Escalante B, Abraham NG, McGiff J C, Levere RD \& Schwartzman $M L$ (1989). Treatment with tin prevents the development of hypertension in spontaneously hypertensive rats. Science, 243 : 388-390.

32. Tenhunen R, Tokola O \& Linden IB (1987). Haem arginate: a new stable haem compound. J ournal of Pharmacy and Pharmacology, 39: 780-786.

33. Linden I-B, Tokola O, Karlsson $M \&$ Tenhunen R (1987). Fate of heme after parenteral administration of heme arginate to rabbits. J ournal of Pharmacy and Pharmacology, 39: 96-102.

34. Vallier HA, Rodgers PA \& Stevenson DK (1993). Inhibition of heme oxygenase after oral vs intraperitoneal administration of chromium porphyrins. Life Sciences, 52: 79-84.
35. Vreman HJ , Ekstrand BC \& Stevenson DK (1993). Selection of metalloporphyrin heme oxygenase inhibitors based on potency and photoreactivity. Pediatric Research, 33: 195-200.

36. Ignarro LJ , Wood KS \& Wolin MS (1984). Regulation of purified soluble guanylate cyclase by porphyrins and metalloporphyrins: a unifying concept. Advances in Cyclic Nucleotide and Protein Phosphorylation Research, 17: 267-274.

37. Grundemar L \& Ny L (1997). Pitfalls in using metalloporphyrins in carbon monoxide research. Trends in Pharmacological Sciences, 18: 193-195.

38. Kanten WE, Penney DG, Francisco K \& Thill J E (1983). Hemodynamic response to acute carboxyhemoglobinemia in the rat. American J ournal of Physiology, 244: $\mathrm{H} 320-\mathrm{H} 327$.

39. Levere RD, Martasek $P$, Escalante $B$, Schwartzman ML \& Abraham NG (1990). Effect of heme arginate administration on blood pressure in spontaneously hypertensive rats. J ournal of Clinical Investigation, 86: 213-219.

40. J ohnson RA, Lavesa M, Askari B, Abraham NG \& Nasjletti A (1995). A heme oxygenase product, presumably carbon monoxide, mediates a vasodepressor role in rats. Hypertension, 25: 166-169.

41. Verma A, Hirsch DJ , Glatt CE, Ronnett GV \& Snyder SH (1993). Carbon monoxide: A putative neural messenger. Science, 259: 381-384.

42. Zakhary R, Gaine SP, Dinerman J L, Ruat M, Flavahan NA \& Snyder SH (1996). Heme oxygenase 2: endothelial and neuronal localization and role in endotheliumdependent relaxation. Proceedings of the National Academy of Sciences, USA, 93: 795-798.

43. J ohnson RA, Colombari E, Colombari DSA, Lavesa M, Talman WT \& Nasjletti A (1997). Role of endogenous carbon monoxide in central regulation of arterial pressure. Hypertension, 30: 962-967.

44. Talman WT \& Reis DJ (1994). Brain lesions and hypertension. In: Ganten G \& de J ong W (Editors), Handbook of Hypertension: Experimental and Genetic Models of Hypertension. Vol. 19. Elsevier, Amsterdam, Holland.

45. Colombari E, Bonagamba LG \& Machado $\mathrm{BH}$ (1994). Mechanisms of pressor and bradycardic responses to L-glutamate microinjected into the NTS of conscious rats. American J ournal of Physiology, 266: R730-R738.

46. Glaum SR \& Miller RJ (1993). Zinc-protoporphyrin-IX blocks the effects of metab- 
otropic glutamate receptor activation in the rat nucleus tractus solitarii. Molecular Pharmacology, 43: 965-969.

47. Colombari E, Silva CCS, Almeida VA, Haibara AS \& J ohnson RA (1998). Heme oxygenase inhibitor (ZnDPG) microinjected into the nucleus tractus solitarii (NTS) affects the baroreflex and the cardiovascular responses induced by L-glutamate (L-glu) in awake rats. FASEB J ournal, 12: 61(Abstract).

48. Grozdanovic Z \& Gossrau R (1996). Expression of heme oxygenase-2 ( $\mathrm{HO}-2$ )-like immunoreactivity in rat tissues. Acta Histochemica, 98: 203-214.

49. Matsuoka A, Stuehr DJ , Olson J S, Clark P \& Ikeda-Saito M (1994). L-arginine and calmodulin regulation of the heme iron reactivity in neuronal nitric oxide synthase. J ournal of Biological Chemistry, 269: 20335-20339.

50. Pufahl RA \& Marletta MA (1993). Oxida- tion of NG-hydroxy-L-arginine by nitric oxide synthase: evidence for the envolvement of the heme in catalysis. Biochemical and Biophysical Research Communications, 193: 963-970.

51. McMillan K \& Masters BSS (1995). Prokaryotic expression of the heme- and flavin-binding domains of rat neuronal nitric oxide synthase as distinct polypeptides: Identification of the heme-binding proximal thiolate ligand as cysteine-415. Biochemistry, 34: 3686-3693.

52. Klatt $P$, Schmidt $K \&$ Mayer B (1992). Brain nitric oxide synthase is a haemoprotein. Biochemical J ournal, 288: 15-17.

53. Kozma F, J ohnson RA \& Nasjletti A (1997). Role of carbon monoxide in heme-induced dilation. European J ournal of Pharmacology, 323: R1-R2.

54. Coceani F, Kelsey L, Seidlitz E, Marks GS, McLaughlin BE, Vreman HJ, Stevenson DK, Rabinovitch M \& Ackerley C (1997).
Carbon monoxide formation in the ductus arteriosus in the lamb: implications for the regulation of muscle tone. British J ournal of Pharmacology, 120: 599-608.

55. Suematsu M, Kashiwagi S, Sano T, Goda N, Shinoda Y \& Ishimura Y (1994). Carbon monoxide as an endogenous modulator of hepatic vascular perfusion. Biochemical and Biophysical Research Communications, 205: 1333-1337.

56. Suematsu M, Goda N, Sano T, Kashiwagi S, Egawa T, Shinoda $Y$ \& Ishimura $Y$ (1995). Carbon monoxide: an endogenous modulator of sinusoidal tone in the perfused rat liver. J ournal of Clinical Investigation, 96: 2431-2437.

57. Kozma F, Ligeti L, Monos E, Colombari E \& J ohnson RA (1998). Heme-derived CO: Endogenous regulator of nitric oxide synthase? Pathophysiology, 5 (Suppl 1): 245 (Abstract). 